\title{
Robustly Optimal Instrument Rules and Robust Control: An Equivalence Result
}

\author{
Carl E. Walsh* \\ Final version: May 2004
}

\begin{abstract}
Giannoni and Woodford (2003a, 2003b) and Hansen and Sargent (2003, 2004) have recently developed different notions of robust policy, and both approaches have been applied in the context of optimal monetary policy. In this note, I demonstrate that both approaches lead to exactly the same implicit instrument rule for the policy maker in a standard, forward-looking, new Keynesian model. Despite the equivalence of the policy rules, the two approaches predict different macroeconomic behavior because of difference assumptions about the formation of expectations.
\end{abstract}

\section{Introduction}

The recent literature on optimal monetary policy in forward-looking models has explored various notions of robustness. For example, Giannoni and Woodford (2003a, 2003b), building on earlier work by Svensson and Woodford (2004), have proposed what they call robustly optimal instrument rules. ${ }^{1}$ These instrument rules do not depend on the specification of the generating process of the exogenous disturbances in

*Professor, Department of Economics, University of California, Santa Cruz, walshc@ucsc.edu. I would like to thank Richard Dennis, Federico Ravenna and John C. Williams for very useful discussions and the referee for helpful comments.

${ }^{1}$ These policy rules are also called targeting rules. See Woodford and Svensson (2004). 
the model. The rules are, therefore, robust to mis-specifications in these generating processes.

In a series of papers, Hansen and Sargent, together with coauthors, have explored robust control approaches to the decision problem of agents who face model uncertainty (Hansen and Sargent 2003, 2004). Robust policies, in the sense of Hansen and Sargent, are designed to perform well in worst-case scenarios. These policies arise as the equilibrium in a game between the policy maker and an evil agent who chooses model mis-specification to make the policy maker look as bad as possible.

While the Giannoni and Woodford and the Hansen and Sargent approaches to robust policies appear quite distinct, in this note I demonstrate that both approaches lead to exactly the same implicit instrument rule for the policy maker in a standard, forward-looking, new Keynesian model of optimal monetary policy.

\section{The robustly optimal rule}

The benchmark new Keynesian model consists of two key structural relationships. The first equation is derived from the first order Euler condition that arises from the representative household's optimal consumption decision. It relates the output gap $x_{t}$ to its expected future value and the real interest rate gap, the difference between the actual real interest rate and the natural real rate $r_{t}^{n}$ :

$$
x_{t}=E_{t} x_{t+1}-\left(\frac{1}{\sigma}\right)\left(i_{t}-E_{t} \pi_{t+1}-r_{t}^{n}\right) .
$$

For simplicity, assume $r_{t}^{n}$ is exogenous and evolves according to

$$
r_{t}^{n}=\rho_{r} r_{t-1}^{n}+\chi_{t}, 0 \leq \rho_{r}<1,
$$

where the innovation $\chi_{t}$ is white noise. The second structural relationship is an inflation adjustment equation that arises in the presence of monopolistic competition 
and a Calvo model of sticky nominal prices:

$$
\pi_{t}=\beta E_{t} \pi_{t+1}+\kappa x_{t}+e_{t}
$$

The cost shock $e_{t}$ captures any factors affecting inflation that alter the relationship between real marginal cost and the output gap. Assume

$$
e_{t}=\rho_{e} e_{t-1}+\varepsilon_{t}, 0 \leq \rho_{e}<1
$$

The innovation $\varepsilon_{t}$ is white noise. ${ }^{2}$

As in Giannoni and Woodford (2003b), the central bank's objective is to minimize a loss function that depends on the variation in inflation, the output gap, and the nominal interest rate:

$$
J_{t}=\left(\frac{1}{2}\right) E_{t} \sum_{i=0}^{\infty} \beta^{i}\left[\pi_{t+i}^{2}+\lambda_{x} x_{t+i}^{2}+\lambda_{i}\left(i_{t+i}-i^{*}\right)\right]
$$

Woodford (2003) discusses the conditions under which (5) can be interpreted as a second order approximation to the welfare of the representative agent.

The optimal commitment policy in the model consisting of (1) - (5) has been studied extensively (Svensson and Woodford 2004, Giannoni and Woodford 2003b, Woodford 2003). The Lagrangian for this decision problem is

$$
\begin{aligned}
L_{t}= & \mathrm{E}_{t} \sum_{i=0}^{\infty} \beta^{i}\left[\left\{\left(\frac{1}{2}\right) \pi_{t+i}^{2}+\left(\frac{1}{2}\right) \lambda_{x} x_{t+i}^{2}+\left(\frac{1}{2}\right) \lambda_{i}\left(i_{t+i}-i^{*}\right)^{2}\right.\right. \\
& s_{1 t+i}\left(\pi_{t+i}-\beta \pi_{t+i+1}-\kappa x_{t+i}-e_{t+i}\right) \\
& \left.+s_{2 t+i}\left[x_{t+i}-x_{t+i+1}+\left(\frac{1}{\sigma}\right)\left(i_{t+i}-\pi_{t+i+1}-r_{t+i}^{n}\right)\right]\right\},
\end{aligned}
$$

where $s_{1}$ and $s_{2}$ denote Lagrangian multipliers. The first order conditions for inflation, the output gap, and the nominal rate of interest take the form

\footnotetext{
${ }^{2}$ See Walsh (2003, Chapter 5) for further discussion and references.
} 


$$
\begin{gathered}
\pi_{t}+s_{1 t}-s_{1 t-1}-\left(\frac{1}{\beta \sigma}\right) s_{2 t-1}=0 \\
\lambda_{x} x_{t}-\kappa s_{1 t}+s_{2 t}-\left(\frac{1}{\beta}\right) s_{2 t-1}=0, \\
\lambda_{i}\left(i_{t}-i^{*}\right)+\left(\frac{1}{\sigma}\right) s_{2 t}=0 .
\end{gathered}
$$

Under the fully optimal commitment policy, the multipliers $s_{1 t-1}$ and $s_{2 t-1}$ are set to zero, as no previous commitments are binding in the first period. However, Woodford has argued for adopting a "timeless perspective" in defining optimal policy in which these multipliers satisfy the same first order conditions as the current and future multipliers do. Specifically, assume the optimal commitment policy from a timeless perspective has been in place since at least $t-2$. This assumption allows one to use (8) to eliminate $s_{2 t}$ and $s_{2 t-1}$ from (7) and then use the resulting expression for $s_{1}$ to eliminate $s_{1 t}$ and $s_{1 t-1}$ from (6). Doing so yields an expression for the nominal rate of interest of the form

$$
i_{t}=-\left(\frac{\kappa}{\sigma \beta}\right) i^{*}+\left(1+\frac{\kappa}{\sigma \beta}+\frac{1}{\beta}\right) i_{t-1}-\left(\frac{1}{\beta}\right) i_{t-2}+\left(\frac{\kappa}{\sigma \lambda_{i}}\right) \pi_{t}+\left(\frac{\lambda_{x}}{\sigma \lambda_{i}}\right)\left(x_{t}-x_{t-1}\right)
$$

This is the Giannoni-Woodford robustly optimal instrument rule. As they emphasize, the coefficients in (9) do not depend on either the serial correlation coefficients $\rho_{r}$ and $\rho_{e}$ or on the variances of the innovations to the disturbances. It is, therefore, robust with respect to mis-specification of these aspects of the model. ${ }^{3}$

\section{Robust control}

The policy problem leading to the optimal commitment policy given by (9) assumed the central bank knew the model with certainty, with the only uncertainty arising from the unknown future realizations of the exogenous shock innovation $\varepsilon$ and $\chi$. In

\footnotetext{
${ }^{3}$ The disturbances do not need to be restricted to the $A R(1)$ structure assumed in (2) and (4). However, they are restricted to be stationary, stochastic processes.
} 
the robust control approach developed by Hansen and Sargent (2004), the central bank views its model as an approximation to the true model of the economy, knowing only that the true model is in a neighborhood around its approximating model.

As Hansen and Sargent (2004) explain, the policy maker's problem can be represented as a game between the policy maker who attempts to minimize the loss function (5) and nature (or an evil agent) who tries to maximize the same loss function. A robust policy is designed to perform well in this worst-case scenario. Hansen and Sargent show how this game can be represented in what they label the multiplier version of the robust Stackleberg problem. This problem takes the form (see Hansen and Sargent 2004 Ch. 15)

$$
\begin{aligned}
& \min _{i} \max _{w_{1}, w_{2}} E_{t}^{r c} \sum_{i=0}^{\infty} \beta^{i}\left[\left(\frac{1}{2}\right) \pi_{t+i}^{2}+\left(\frac{1}{2}\right) \lambda_{x} x_{t+i}^{2}+\left(\frac{1}{2}\right) \lambda_{i}\left(i_{t+i}-i^{*}\right)^{2}\right. \\
& -\left(\frac{1}{2}\right) \beta \theta\left(w_{1 t+1+i}^{2}+w_{2 t+i+1}^{2}\right)+s_{1 t+i}\left(\pi_{t+i}-\beta \pi_{t+i+1}-\kappa x_{t+i}-e_{t+i}\right) \\
& +s_{2 t+i}\left[x_{t+i}-x_{t+i+1}+\left(\frac{1}{\sigma}\right)\left(i_{t+i}-\pi_{t+i+1}-r_{t+i}^{n}\right)\right] \\
& +s_{3 t+i}\left(\rho_{e} e_{t+i}+\varepsilon_{t+i+1}+w_{1 t+i+1}-e_{t+i+1}\right) \\
& \left.+s_{4 t+i}\left(\rho_{r} r_{t+i}^{n}+\chi_{t+i+1}+w_{2 t+i+1}-r_{t+i+1}^{n}\right)\right] .
\end{aligned}
$$

The new variables $w_{1 t+i}$ and $w_{2 t+i}$ represent the model mis-specifications that enter into the dynamics of the model's state variables. The new parameter $\theta$ reflects the policy maker's desired degree of robustness, with $0<\theta<\infty$. The standard rational expectations model arises when $\theta=\infty$.

The superscript $r c$ is added to the expectations operator to reflect the assumption about the policy maker's and the public's expectations that is implicit in this formulation. In the standard rational expectations framework employed in section 2, private agents and the policy maker are assumed to share the same model and information, so the expectations of both coincide. Paralleling this assumption, I following Hansen and Sargent (2004, ch. 15) and Giordani and Söderlind (2003) and assume private agents share the policy maker's approximating model and degree of robustness. In this situation, the public's and the policy maker's expectations about future 
inflation and output coincide. Because expectations will incorporate the behavior of the evil agent, they will differ from rational expectations based on the approximating model.

The assumption of common expectations is clearly not the only possible one. An alternative approach would be to assume private agents are concerned about robustness but have a desired degree of robustness that differs from that of the policy maker. Hansen and Sargent (2003) analyze this case in a model of a monopolist with a competitive fringe. As they show, the monopolist must incorporate the representative competitive firm's worst case beliefs into its decision problem. An interesting area for future research would be to allow private agents and the policy maker to have different worst case models. However, If the policy maker's objective is to maximize the expected welfare of the representative agent, then it would seem natural to assume the policy maker's behavior reflects the same desire for robustness as that of private agents.

Again assuming a timeless perspective, the first order conditions with respect to $\pi_{t}, x_{t}, i_{t}, e_{t}, r_{t}^{n}, w_{1 t}$, and $w_{2 t}$ for the robust Stackleberg problem are

$$
\begin{gathered}
\pi_{t}+s_{1 t}-s_{1 t-1}-\left(\frac{1}{\beta \sigma}\right) s_{2 t-1}=0, \\
\lambda_{x} x_{t}-\kappa s_{1 t}+s_{2 t}-\left(\frac{1}{\beta}\right) s_{2 t-1}=0 \\
\lambda_{i}\left(i_{t}-i^{*}\right)+\left(\frac{1}{\sigma}\right) s_{2 t}=0, \\
-s_{1 t}+\rho_{e} s_{3 t}-\left(\frac{1}{\beta}\right) s_{3 t-1}=0, \\
-\left(\frac{1}{\sigma}\right) s_{2 t}+\rho_{r} s_{4 t}-\left(\frac{1}{\beta}\right) s_{4 t-1}=0, \\
-\theta w_{1 t+1}+s_{3 t}=0 \\
-\theta w_{2 t+1}+s_{4 t}=0 .
\end{gathered}
$$

Note that equations (10) - (12) are identical to the first order conditions (6) - 
(8). Thus, proceeding as before, the Lagrangian multipliers $s_{1}$ and $s_{2}$ can be eliminated from these equations to yield an implicit instrument rule for the nominal rate of interest that is identical to (9). This establishes the equivalence between the policy rule under the robustly optimal (timeless) commitment policy and under the optimal commitment policy when the policy maker has a desire for robustness. Note that the coefficients in this rule are independent of $\theta$, the policy maker's desired degree of robustness.

The robustly optimal rule is designed to be robust to mis-specification of the process generating the exogenous disturbances. The robust control approach considers policies that are robust to mis-specification of the equations characterizing the evolution of the state variables. But since in the basic new Keynesian model these state variables are just the disturbances, both approaches are designed to yield policies that are robust to the same sources of mis-specification. Both, therefore, lead to the same implicit instrument rule.

\section{A specific example: inflation targeting}

Despite the fact that the robustly optimal and the robust control policy rules are identical, the economy behaves differently under the two model specifications. This can be illustrated in a simplified version of the model, obtained by setting $\lambda_{i}=0$. In this case, the central bank's loss function involves only inflation and the output gap. This specification yields the most commonly used form of the loss function, one often identified with an inflation targeting regime. As is well known, equation (1) becomes redundant when the nominal interest rate does not appear in the loss function, and the output gap itself can be treated as the direct instrument of monetary policy.

Using (6) and (7) and setting $s_{2}=0$, the behavior of the economy under the optimal commitment policy (timeless perspective) is characterized by

$$
\pi+s_{1 t}-s_{1 t-1}=0
$$


and

$$
\lambda_{x} x_{t}-\kappa s_{1 t}=0
$$

together with the inflation adjustment equation (3) and

$$
e_{t}=\rho_{e} e_{t-1}+\varepsilon_{t}
$$

Combining the first two of these equations and using (3), one obtains

$$
\left[1+\beta+\left(\frac{\kappa^{2}}{\lambda_{x}}\right)\right] x_{t}=\beta E_{t} x_{t+1}+x_{t-1}-\left(\frac{\kappa}{\lambda_{x}}\right) e_{t},
$$

which can be jointly solved with the process for $e_{t}$ given by (17) under rational expectations.

Under robust control, the first order conditions become

$$
\begin{gathered}
\pi+s_{1 t}-s_{1 t-1}=0, \\
\lambda_{x} x_{t}-\kappa s_{1 t}=0, \\
-s_{1 t}+\rho_{e} s_{3 t}-\left(\frac{1}{\beta}\right) s_{3 t-1}=0,
\end{gathered}
$$

and

$$
-\theta w_{1 t+1}+s_{3 t}=0
$$

together with the inflation adjustment equation

$$
\pi_{t}=\beta E_{t}^{r c} \pi_{t+1}+\kappa x_{t}+e_{t}
$$

and

$$
e_{t+1}=\rho_{e} e_{t}+\varepsilon_{t+1}+w_{1 t+1} .
$$

The presence of $w_{1}$ in (24) reflects the possible model mis-specification. 
Combining (19), (20), and (23) yields

$$
\left[1+\beta+\left(\frac{\kappa^{2}}{\lambda_{x}}\right)\right] x_{t}=\beta E_{t}^{r c} x_{t+1}+x_{t-1}-\left(\frac{\kappa}{\lambda_{x}}\right) e_{t},
$$

which is identical to (18), except for the formation of expectations.

Equations (21) and (20) imply that

$$
s_{3 t-1}=\beta \rho_{e} s_{3 t}-\beta s_{1 t}=\beta \rho_{e} s_{3 t}-\beta\left(\frac{\lambda_{x}}{\kappa}\right) x_{t} .
$$

Advancing this expression one period, taking expectations, solving the resulting expression forward, and using (22) implies that

$$
w_{1 t+1}=-\left(\frac{\beta \lambda_{x}}{\kappa \theta}\right) \sum_{i=0}^{\infty}\left(\beta \rho_{e}\right)^{i} E_{t}^{r c} x_{t+1+i}
$$

Model mis-specification is related to the present value of the expected output gap. If the output gap is expected to be negative, $w_{1}$ is positive, acting like a positive cost shock that raises inflation. Because cost shocks present the policy maker with a trade-off between inflation stabilization and output gap stabilization, a worst-case scenario for the policy maker when the output gap is already negative occurs when a positive cost shock hits the economy. This forces the policy maker to further contract output to offset the inflationary impact of the shock. This worst-case is reflected in the policy maker's distorted model; future cost shocks are expected to be negatively related to a discounted sum of future output gaps.

Substituting (26) into (24) yields the distorted process for the cost shock. Because expectations are based on this distorted process, one distorted to reflect the worst-case situation for the policy maker, the solution for the output gap obtained from (25), (24), and (26) will differ from that obtained in the standard case from equations (18) and (17). 


\section{Extensions}

This section very briefly considers two extensions to the analysis.

\subsection{Lagged endogenous state variables}

In the version of the new Keynesian model employed in section 2, no lagged endogenous state variables appeared. It is common, however, to incorporate lagged inflation into the inflation adjustment equation to correct what many consider to be the poor empirical performance of the purely forward-looking inflation equation. It is straightforward to show that the equivalence result continues to hold in this case. The transition equations for the exogenous disturbances remain the only ones affected by the evil agent. The new transition equations that appear in the state space form of the model simply keep track of the fact that today's inflation becomes tomorrow's lagged inflation; this relationship is definitional and therefore not subject to distortion by the evil agent.

\subsection{A simple rule for the evil agent ${ }^{4}$}

In section 3, the evil agent commits to an optimal policy. An alternative approach would treat the evil agent as committing to a simple rule. This case is of interest because it illustrates very clearly how the desire for robustness on the part of the policy maker leads to a distorted process for the cost shock. Since the Giannoni-Woodford robustly optimal rule is designed to be independent of the cost shock process, it remains optimal even in the presence of the evil agent.

Suppose the evil agent chooses a contingency rule for the $w_{t+1}^{\prime} s$ that makes them functions of exogenous state variables. Specifically, in the version of the model with $\lambda_{i}=0$, assume that the evil agent commits to setting $w_{1 t+1}=K e_{t}$. Define $\tilde{\rho}_{e}=\rho_{e}+K$. Then, for any choice of $K$ such that $\left|\tilde{\rho}_{e}\right|<1$, the policy maker's

\footnotetext{
${ }^{4}$ I would like to thank the referee for suggesting this case.
} 
problem becomes

$$
\begin{aligned}
& \min _{i} E_{t}^{r c} \sum_{i=0}^{\infty} \beta^{i}\left\{\left(\frac{1}{2}\right) \pi_{t+i}^{2}+\left(\frac{1}{2}\right) \lambda_{x} x_{t+i}^{2}-\left(\frac{1}{2}\right) \beta \theta\left(F e_{t+i}\right)^{2}\right. \\
& +s_{1 t+i}\left(\pi_{t+i}-\beta \pi_{t+i+1}-\kappa x_{t+i}-e_{t+i}\right) \\
& \left.+s_{3 t+i}\left(\tilde{\rho}_{e} e_{t+i}+\varepsilon_{t+i+1}-e_{t+i+1}\right)\right\} .
\end{aligned}
$$

Note that this is a standard problem with the shock process (4) for the approximating model replaced by the distorted process

$$
e_{t+1}=\tilde{\rho}_{e} e_{t}+\varepsilon_{t+1}
$$

The policy maker (and the public) takes $\tilde{\rho}_{e}$ (i.e., $K$ ) as given. Treating $x_{t}$ as the instrument of policy (since $\lambda_{i}=0$ ), the robustly optimal instrument rule is $x_{t}=x_{t-1}-\left(\kappa / \lambda_{x}\right) \pi_{t}$, which is independent of $\tilde{\rho}_{e}$ (and therefore $K$ ). This independence reflects the fact that the robustly optimal instrument rule of Giannoni and Woodford is designed to be robust with respect to exactly the type of model mis-specification of the disturbance process that is reflected in (27).

\section{Conclusions}

Two seemingly independent strands in the recent literature on optimal monetary policy in forward-looking models lead to identical policy rules. Under both approaches, the policy makers maintains the same relationship between its instrument, inflation, and the output gap. Despite the equivalence of the policy rules, the two approaches predict different macroeconomic behavior. This difference arises because expectations are formed differently in the two approaches. 


\section{References}

[1] Giannoni, Marc and Michael Woodford. (2003a) "Optimal Interest-Rate Rules: I. General Theory." NBER Working Paper No. 9419.

[2] Giannoni, Marc and Michael Woodford. (2003b) "Optimal Interest-Rate Rules: II. Applications." NBER Working Paper No. 9420.

[3] Hansen, Lars Peter and Thomas J. Sargent. (2003) "Robust Control of ForwardLooking Models," Journal of Monetary Economics, 50, 581-604.

[4] Hansen, Lars Peter and Thomas J. Sargent. (2004) Robust Control and Economic Model Uncertainty. Princeton, NJ: Princeton University Press.

[5] Svensson, Lars E. O. and Michael Woodford. (2004) "Implementing Optimal Policy through Inflation-Forecast Targeting." In Ben S. Bernanke and Michael Woodford (ed.), The Inflation Targeting Debate. Chicago: University of Chicago Press. Forthcoming.

[6] Walsh, Carl E. (2003) Monetary Theory and Policy, 2nd. ed. Cambridge, MA: The MIT Press.

[7] Woodford, Michael. (2003) Interest and Prices. Princeton, NJ: Princeton University Press. 